\title{
Optical bistability by two-wave mixing in photorefractive crystals
}

\author{
Edward D. Baraban \\ Eastman Kodak Company, Rochester, New York 14653, and The Institute of Optics, University of Rochester, \\ Rochester, New York 14627 \\ Hong-Zhun Zhang \\ Institute of Physics, Beijing, China, and The Institute of Optics, University of Rochester, Rochester, New York 14627
}

\author{
Robert W. Boyd
}

The Institute of Optics, University of Rochester, Rochester, New York 14627

Received January 8, 1992

Optical bistability in photorefractive strontium barium niobate in a two-wave mixing configuration with feedback is reported. We observe bistability both under conditions for which its existence was recently predicted by Zozulya et al. [Opt. Commun. 72, 381 (1989)] and under more general conditions.

Optical bistability and switching are of great interest in the rapidly developing field of photonics. Devices that display this behavior could potentially play a major role in the development of optical communication systems and optical computing. Bistability in photorefractive crystals has been observed in a semilinear self-pumped configuration, ${ }^{1}$ a ring resonator configuration, ${ }^{2-4}$ and a totalinternal-reflection self-pumped configuration., ${ }^{5,6}$ Each of these devices operates by means of a four-wave mixing process. Recently, however, bistability in photorefractive two-wave mixing was predicted theoretically. ${ }^{7-9}$

Here we present the first published experimental observation to our knowledge of optical bistability in a photorefractive crystal by two-wave mixing with feedback. Our study was motivated by theoretical predictions made by Zozulya et $a l^{9}$ We observe bistability as predicted by this theory; however, we also observe bistability under conditions for which the presence of bistability is not predicted by this theory. In each case we observe bistability both when the feedback is provided by an electronic system and when the feedback is provided by an all-optical system.

Let two coherent, monochromatic optical waves intersect within a photorefractive crystal of length $l$ as shown in the inset in Fig. 1. A refractive-index grating is written by the mixing of waves $I_{1}$ and $I_{2}$. One of the transmitted waves, $I_{2}$, is fed back into the crystal with reflectivity $r$ as an erase beam; it does not interact with $I_{1}$ or $I_{2}$ to form additional gratings. The set of equations that describes the interaction of the waves in this sytem is given by ${ }^{10}$

$$
\begin{gathered}
\frac{\partial A_{1}}{\partial x}=\nu A_{2}, \\
\frac{\partial A_{2}^{*}}{\partial x}=-\nu A_{1}^{*},
\end{gathered}
$$

$$
\left(\tau_{d} \frac{\partial}{\partial t}+1\right) \nu=\frac{\gamma}{I_{0}} A_{1} A_{2}^{*}
$$

where $\nu$ is the amplitude of the photorefractive grating, $I_{j} \equiv\left|A_{j}\right|^{2}$,

$$
I_{0}=I_{1}(0, t)+I_{2}(0, t)+r I_{2}(l, t),
$$

$\tau_{d}$ is the photorefractive response time, and $\gamma$ is a nonlinear coupling coefficient that in the simplest approximation is dependent only on crystal material parameters and geometry.

For steady-state conditions we can obtain a closed-form solution to Eqs. (1)-(3), given by ${ }^{9}$

$$
G\left[1+m \exp \left(\frac{2 \gamma l}{1+r G}\right)\right]=1,
$$

where $G$ is the normalized transmitted intensity for beam 2 , given by

$$
G=\frac{i_{2}(l)}{I_{1}(0)+I_{2}(0)}
$$

and $m$ is the modulation index of the input beams, given by

$$
m=\frac{I_{1}(0)}{I_{2}(0)}
$$

A plot of Eq. (5) is shown in Fig. 1 for the coupling constant $\gamma l=3$ and for several values of the feedback reflectivity $r$. Solid curves indicate stable branches, and dashed curves indicate unstable branches. Note that bistability occurs for a large range of values of $r$ and $\gamma l$ but only for $r>1$ and for $\gamma E l>0$. These conditions imply that the optical components used to produce the erase 


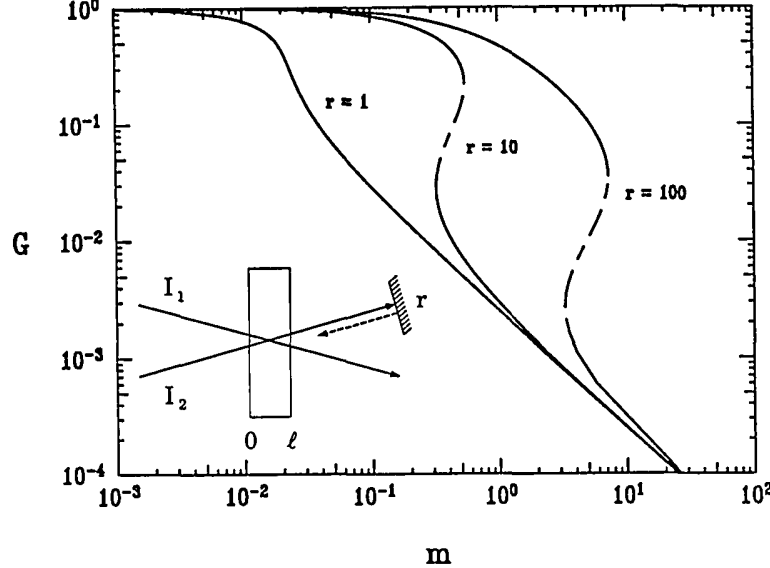

Fig. 1. Plot of Eq. (6) for $y l=3$ with $r$ equal to 1, 10, and 100 and (inset) schematic diagram of bistability by two-wave mixing with feedback, where $m=I_{1} / I_{2}$.

beam must provide amplification and that the direction of energy transfer in the photorefractive crystal is away from the wave used for feedback, which in this case is $I_{2}$.

Our first observations of bistability involve a hybrid system, shown in Fig. 2(a). An argon-ion laser operating at a wavelength of $514.5 \mathrm{~nm}$ in a single longitudinal mode serves as the coherent light source. The output of the laser is split into a strong beam $(\sim 20 \mathrm{~mW})$, which is directed to a Pockels cell, and two weak beams $(\sim 2 \mathrm{~mW})$, which intersect within a photorefractive crystal. The crystal used for all the bistability experiments is a $15 \mathrm{~mm} \times 7 \mathrm{~mm} \times 1.85 \mathrm{~mm}$ sample of strontium barium niobate (SBN) doped with $0.1 \%$ cerium. The $c$ axis of the crystal is oriented such that it is in the plane of the figure and parallel to the $15-\mathrm{mm}$ side of the sample. For any particular geometry, $\gamma l$ was measured by using the relation (valid for small modulation depths) ${ }^{11}$

$$
\gamma l=\ln \frac{I_{\text {signal }}^{\prime} I_{\text {pump }}}{I_{\text {signal }} I_{\text {pump }}^{\prime}}
$$

where the primed symbols denote intensities measured in the presence of coupling and the unprimed symbols denote intensities measured in the absence of coupling.

After passing through the crystal, a fraction of $I_{2}$ is directed to detector $\mathrm{D} 1$, which is used to monitor the transmitted intensity $I_{2}(l)$, and the remaining portion of $I_{2}$ is sent to detector D2. D2 produces a voltage that is linearly proportional to $I_{2}(l)$; this voltage is then amplified and sent to the Pockels cell. This modulator controls the intensity of the strong beam passing through it such that the transmitted intensity is always $r I_{2}(l)$. A half-wave plate $(\lambda / 2)$ placed in the path of the feedback beam rotates the beam's polarization by $90^{\circ}$, thus ensuring that it cannot interfere with the mixing waves to form additional gratings.

The use of a thin crystal is essential to the successful operation of these bistable systems. As a light wave passes through a photorefractive crystal, it is subject to stimulated photorefractive scattering. If the amount of scattered light grows significantly over the interaction region, self-pumped phase conjugation can occur. ${ }^{12}$ 'I'his effect will compete with the energy transfer between the mixing waves and tend to suppress the occurrence of bistability.
Another important consideration for the hybrid bistable system is the nature of the response of the Pockels cell. Since, in general, the transmission of a Pockels cell depends nonlinearly on the applied voltage, we have taken great care to operate the cell in a region for which the response is essentially linear. Failure to do so would introduce the possibility of producing bistability as a result of the nonlinearity of the Pockels cell rather than the nonlinearity of the photorefractive response.

Experimental results showing bistable behavior in the hybrid system for $\gamma l=3.02$ and $r=10.1$ are shown in Fig. 3(a). The critical points occur at approximately, but not exactly, the locations predicted by theory. Error bars are not shown, since the fluctuations in the measured signal for any given data point are quite small compared with the size of the hysteresis loop.

The inherent slowness of the photorefractive effect, coupled with the critical slowing phenomenon typically observed in bistable systems, resulted in long datacollection times. As $m$ is increased or decreased to approach a critical point, the system takes several minutes to approach steady state. Close to a critical point the system takes as long as $2 \mathrm{~h}$ to come to its steady-state value.

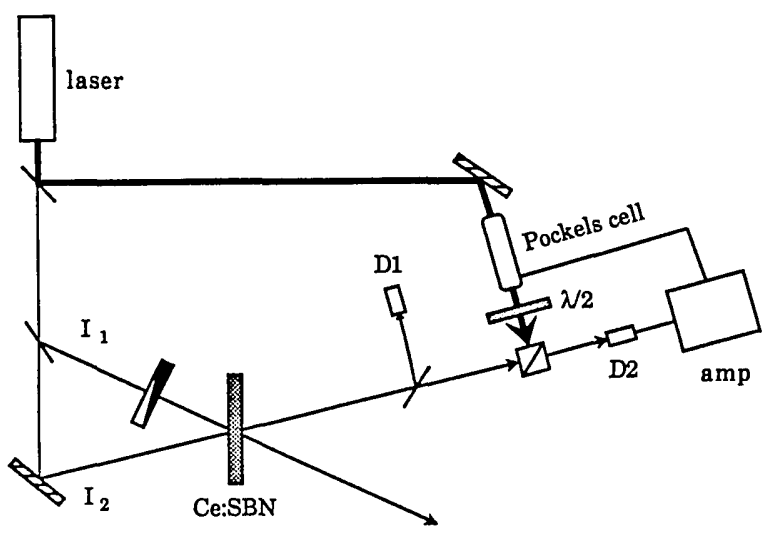

(a)

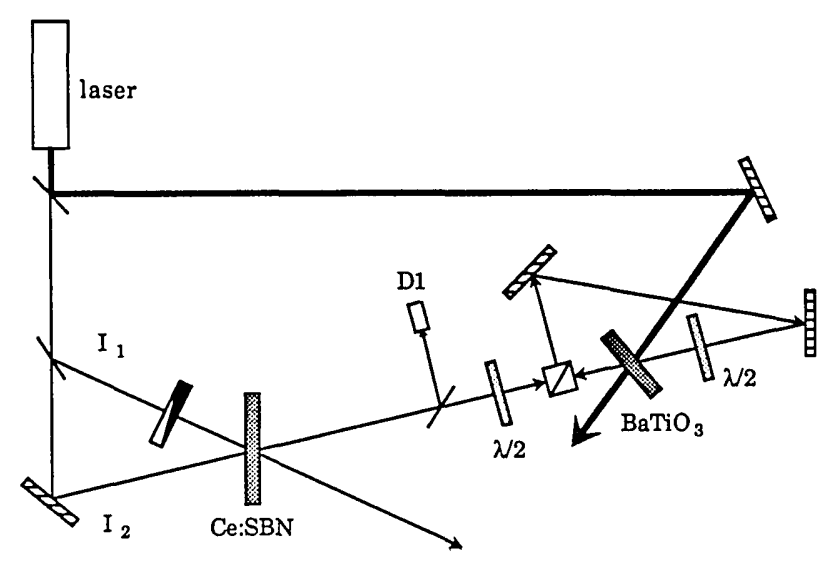

(b)

Fig. 2. Experimental setups used to observe (a) hybrid optical bistability and (b) all-optical bistability. 


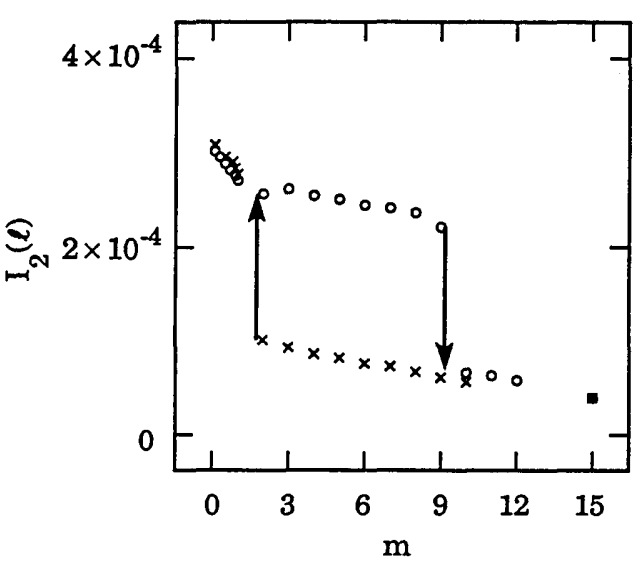

(a)

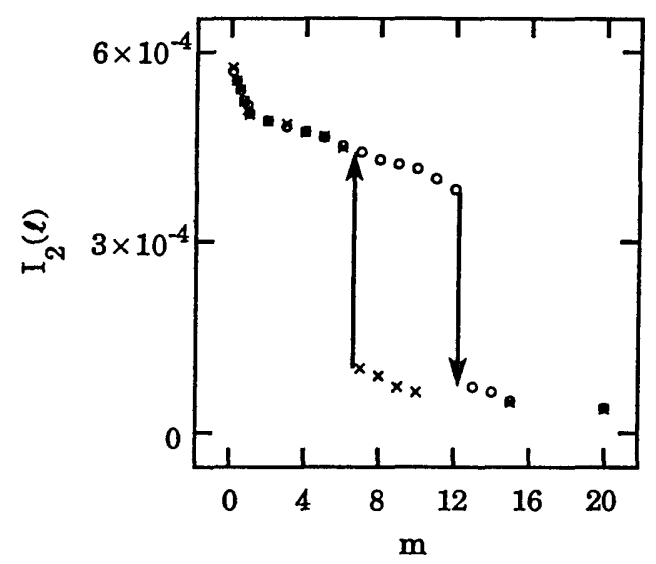

(b)

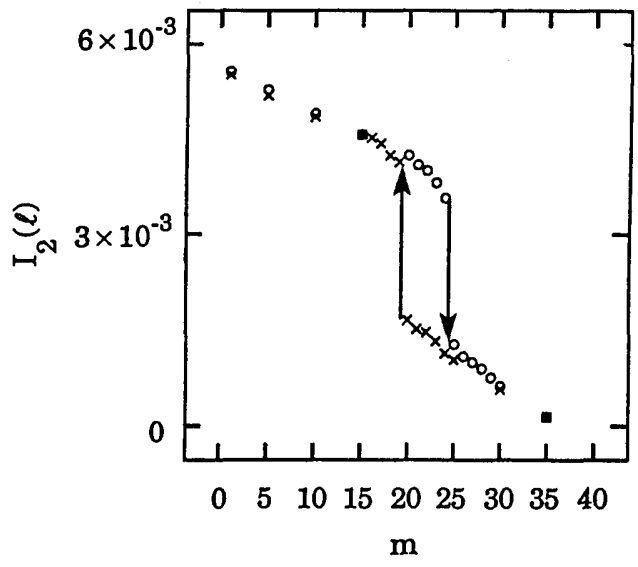

(c)

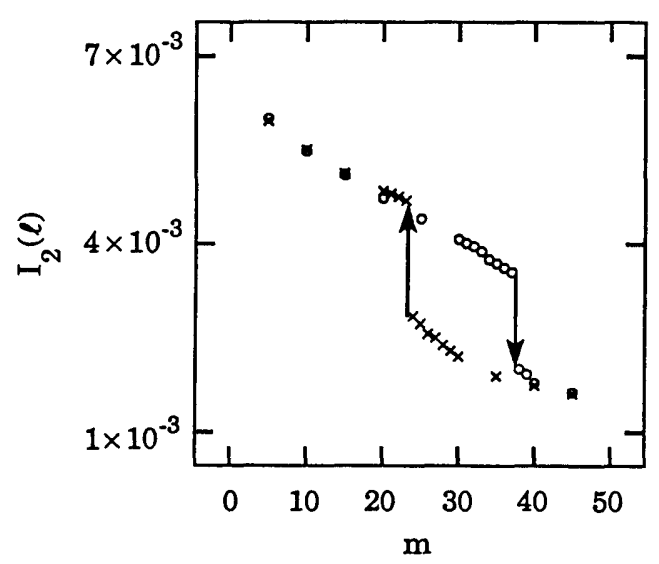

(d)

Fig. 3. Experimental results of (a) hybrid bistability for $\gamma l=3.02$ and $r=10.1$, (b) all-optical bistability for $\gamma l=3.01$ and $r=10$, (c) hybrid bistability for $\gamma l=-2.91$ and $r=12.5$, and (d) all-optical bistability for $\gamma l=-2.91$ and $r=10$.

The exact values of $m$ at the critical points seem to depend sensitively on the mechanical stability of the system. In addition, enclosing the entire apparatus in a box to reduce the flow of air currents through the beam path drastically reduces the noise in the measured signal.

We have also observed optical bistability in an alloptical system. To do so, we replaced the electronically driven components of our setup with a second photorefractive crystal, which served to amplify the feedback beam. This all-optical bistable system is shown in Fig. 2(b). In this case a crystal of barium titanate $\left(\mathrm{BaTiO}_{3}\right)$ with dimensions similar to those of the SBN crystal was used as the amplifying device in the feedback loop. Experimental results showing bistable behavior in the all-optical system for $y l=3.01$ and $r=10$ are shown in Fig. 3(b). These results demonstrate that bistable behavior does not require the presence of electronic components in the system.

Comparison of the experimental results obtained for $\gamma l>0$ and the theory show good qualitative agreement. In general, the critical points of the experimental results occur at higher values of $m$ than do the theoretical results, but these differences presumably can be explained through certain experimental considerations. For example, the theoretical treatment assumes that only a single grating is written by the two mixing waves. In our experimental realization of the model there are probably additional gratings, which could be due, for example, to stimulated photorefractive scattering. In addition, birefringence within the crystal could modify the polarization of the erase beam such that it is not orthogonal to the mixing waves.

We have also performed experiments with the $c$ axis of the SBN crystal rotated by $180^{\circ}$, such that the direction of energy transfer is reversed (i.e., $I_{2}$ is being amplified) and $\gamma l<0$. In this configuration bistability occurs, as is shown in Fig. 3(c) for the hybrid system and Fig. 3(d) for the all-optical system.

In an attempt to explain the occurrence of bistability for the case in which $\gamma l<0$, we have performed numerical integrations of Eqs. (1)-(3). We have found no cases of bistability other than those given by Eq. (5) (with $\gamma l>0$ ).

We have also examined the possibility that bistability can occur for $\gamma l<0$ as a consequence of the nonsinusoidal profile of the photorefractive gratings within the crystal. Equations (1)-(3) presuppose that the grating is purely sinusoidal, which is true only for $m \ll 1$ or $m \gg 1$. In our experiments $m$ was always greater than 15 when bistability was observed for $\gamma l<0$. It is possible that, because of 
the strong photorefractive response of SBN, the photorefractive gratings are appreciably nonsinusoidal even at an intensity ratio of $m=15$. We have modeled this effect by modifying Eq. (3) according to a prescription described by Vachss and Hesselink. ${ }^{13}$ However, we find that, even with the incorporation of this additional nonlinearity, our numerical solutions do not display bistability for $\gamma l<0$.

One possible explanation for the inability of the theory described above to model our experimental results for $\gamma l<0$ is that the theory assumes that the interacting beams are uniform plane waves, whereas the experiment used beams with an approximately Gaussian intensity profile. This difference could be quite significant, because the photorefractive effect depends critically on the relative intensities of the interacting waves.

In summary, we have observed optical bistability by means of photorefractive two-wave mixing in the presence of a feedback erase beam. We observe bistability for $\gamma l>0$, as predicted by the theory presented by Zozulya et al., ${ }^{9}$ but we also observe bistability for $\gamma l<0$, in disagreement with the predictions of this theory. This discrepancy may be due to experimental factors, such as our use of Gaussian laser beams, or it may be due to some fundamental limitations of the standard photorefractive equations.
We are grateful to T. R. Moore for discussions regarding this experiment. This work was supported by the sponsors of the New York State Center for Advanced Optical Technology and by the United States Army Research Office through the University Research Initiative.

\section{REFERENCES}

1. S. Kwong, M. Cronin-Golomb, and A. Yariv, Appl. Phys. Lett. 45, 1016 (1984).

2. S. Kwong and A. Yariv, Opt. Lett. 11, 377 (1986).

3. A. Yariv, S. Kwong, and K. Kyuma, Appl. Phys. Lett. 48, 1114 (1986).

4. H. Jagannath, P. Venkateswarlu, and M. C. George, Opt. Lett. 12, 1032 (1987).

5. R. W. Eason and A. M. C. Smout, Opt. Lett. 12, 51 (1987).

6. J. Rodriquez, A. Siahmakoun, and G. Salamo, Appl. Opt. 26, 2263 (1987).

7. S. Weiss and B. Fischer, Opt. Commun. 70, 515 (1989).

8. S. Weiss and B. Fischer, Opt. Quantum Electron. 22, S17 (1990).

9. A. A. Zozulya, K. Baumgärtel, and K. Sauer, Opt. Commun. 72, 381 (1989).

10. N. V. Kuktarev, V. B. Markov, and S. G. Odulov, Opt. Commun. 23, 338 (1977).

11. N. V. Kuktarev, V. B. Markov, S. G. Odulov, M. S. Soskin, and V. L. Vinetskii, Ferroelectrics 22, 949 (1982).

12. J. Feinberg, Opt. Lett. 7, 486 (1982).

13. F. Vachss and L. Hesselink, J. Opt. Soc. Am. A 5, 690 (1988) 